\title{
Caracterización fisicoquímica, químico proximal, compuestos bioactivos y capacidad antioxidante de pulpa y corteza de sandía (Citrullus lanatus)
}

\author{
Marcelo F. Valle-Vargas(1), Ricardo Durán-Barón ${ }^{(1) \star}$, Greilis Quintero-Gamero(1), y Robert Valera(1) \\ (1) Universidad Popular del Cesar, Sede Sabanas, Grupo de Optimización Agroindustrial, Valledupar, Colombia. \\ (e-mail: mfernandovalle@unicesar.edu.co, ricardoduran@unicesar.edu.co, gquinterog@unicesar.edu.co, \\ agroindustria@unicesar.edu.co) \\ ${ }^{*}$ Autor a quien debe ser dirigida la correspondencia.
}

Recibido May. 24, 2019; Aceptado Jul. 22, 2019; Versión final Sep. 5, 2019, Publicado Feb. 2020

\begin{abstract}
Resumen
Se realizó la caracterización fisicoquímica, químico proximal, compuestos bioactivos (compuestos fenólicos y citrulina) y capacidad antioxidante de pulpa y corteza de tres variedades de sandías (Citrullus lanatus). La caracterización fisicoquímica y químico proximal fueron realizadas mediante los métodos descritos por la Asociación de Químicos Analíticos Oficiales, AOAC. Los compuestos fenólicos, el contenido de citrulina y la capacidad antioxidante fueron cuantificados mediante los métodos de Folin-Ciocalteu, Diacetil monoxima y del radical libre, respectivamente. La pulpa de Crimson Sweet y Santa Amelia presentaron el mayor contenido de compuestos fenólicos y capacidad antioxidante $(61.82 \mathrm{mg} \mathrm{GAE} / 100 \mathrm{~g}$ y $69.30 \mu \mathrm{mol}$ TEAC/100 g de muestra fresca, respectivamente). La corteza de Santa Amelia tuvo el mayor contenido de citrulina $(2.41 \mathrm{mg} / \mathrm{g}$ muestra fresca). La composición de la corteza de sandía muestra que este residuo agroindustrial puede ser usado para la extracción de compuestos bioactivos y su utilización en matrices alimentarias.
\end{abstract}

\section{Physicochemical and proximate chemical characterization, bioactive compounds and antioxidant activity from pulp and rind of watermelons (Citrullus lanatus)}

\begin{abstract}
The physicochemical and proximate chemical characterization, bioactive compounds (phenolic compounds and citrulline) and antioxidant activity of pulp and rind from three watermelon (Citrullus lanatus) cultivars were performed. The physicochemical and proximate chemical characterization were carried out using the methods described by the Association of Official Analytical Chemists, AOAC. Phenolic compounds, citrulline content and antioxidant activity were quantified by the methods of Folin-Ciocalteu, Diacetyl monoxime and free radical, respectively. Crimson Sweet and Santa Amelia pulp showed the highest phenolic content and antioxidant activity (61.82 mg GAE/100 and $69.30 \mu \mathrm{mol}$ TEAC/100 g fresh sample, respectively). Santa Amelia rind had the highest citrulline content $(2.412 \mathrm{mg} / \mathrm{g}$ fresh sample). The composition of watermelon rind showed that this agro-industrial waste could be used for the extraction of bioactive compounds and for its utilization in food matrices.
\end{abstract}

Keywords: watermelon; rind; citrulline; phenolic compounds; antioxidant activity 


\section{INTRODUCCIÓN}

La producción mundial de sandía está concentrada en Asia con un $84.0 \%$, de las cuales los principales productores son China, Irán y Turquía (Kyriacou et al., 2018). En Colombia, entre los años 2010 y 2014 hubo un incremento del $86 \%$ en la producción de sandía; el cual es uno de las cultivos de mayor impacto económico y social por área sembrada (Cohen-Manrique et al., 2018). La sandía es apetecida por muchas personas en el mundo debido a su bajo contenido calórico, su contenido nutricional, sus propiedades sensoriales y por su capacidad para calmar la sed (Tarazona-Díaz et al., 2011). La sandía (Citrullus lanatus) es una fruta grande con forma ovalada o redonda, con piel suave de color verde pálido que se convierte en un verde amarillento cuando el fruto está maduro (Koocheki et al., 2007). La sandía está compuesta por la pulpa (parte comestible de la fruta), las semillas y la cáscara (piel y corteza), esta última representa un $30-40 \%$ del peso total de la fruta, y es considerada un desecho que se utiliza en su mayoría para alimento directo de porcinos; en menor proporción por los amantes a la culinaria para el desarrollo de algunas recetas (Durán et al., 2017). La sandía es una fruta rica en nutrientes como grasa, carbohidratos, fibra, proteínas, minerales y agua, estos componentes están distribuidos en la corteza, pulpa y semilla, principalmente (Fila et al., 2013). Además de estos nutrientes, la sandía (pulpa y corteza) es fuente de compuestos bioactivos tales como compuestos fenólicos, licopeno y citrulina, los cuales al ser ingeridos por el ser humano le proporcionan beneficios a su salud (Tarazona-Díaz et al., 2011). Los compuestos fenólicos muestran actividad antioxidante frente a los radicales libres causantes del estrés oxidativo, atribuyéndoseles a su vez un efecto beneficioso en la prevención de enfermedades tales como: cardiovasculares, circulatorias, cancerígenas y neurológicas (Kuskoski et al., 2005; Tlili et al., 2011).

Entre los compuestos bioactivos presentes en la sandía, la citrulina es de gran importancia, ya que es un aminoácido no esencial de origen no proteico; el cual está presente en mamíferos y también en otros organismos vivos. La citrulina es producida de forma natural por el cuerpo y también se puede encontrar en ciertos alimentos como la sandía, pepino, calabaza, melones, entre otros (Kaore y Kaore, 2014). En el caso de la sandía, la citrulina se puede encontrar tanto en pulpa como en la corteza de la sandía, presentándose en mayor cantidad en esta última (Davis et al., 2011; Rimando y Perkins-Veazie, 2005; Tarazona-Díaz et al., 2011), el cual tiene un fuerte poder antioxidante y es buen eliminador de radicales libres (Al-Sayed y Ahmed, 2013), que tiene la función biológica de ser precursora en la síntesis de L-arginina; aminoácido esencial, que cumple un rol vital en el buen funcionamiento del sistema circulatorio e inmunológico y participa en la producción de Óxido nítrico, que funciona como mensajero celular en el sistema cardiovascular y es una molécula vaso protectora fundamental (Tarazona et al., 2017; Mori et al., 2015). Estas propiedades de la citrulina permiten su acción contra enfermedades como el Alzheimer, anemia de células falciformes, demencia vascular, desorden del ciclo de la urea, diabetes, hipertensión, arterosclerosis, desordenes intestinales, enfermedad celíaca, cáncer, disfunción eréctil, falla renal, problemas respiratorios, entre otras (Kaore y Kaore, 2014).

Cierta parte del residuo (corteza) producido del consumo de sandía es arrojada a tiraderos y rellenos sanitarios provocando un problema medio ambiental (Durán et al., 2017). La valorización de estos residuos es una opción para la reducción de la carga contaminante y una oportunidad para la obtención de compuestos bioactivos como lo son los fenoles y citrulina, los cuales deben ser cuantificados para poder ser incluidos en matrices alimentarias, y así aprovechar sus beneficios en la salud humana (Tarazona-Díaz et al., 2011).Sin embargo, es necesario tener en cuenta que el contenido de compuestos bioactivos en frutas y vegetales puede estar influenciado por varios factores como genotipo de la variedad, condiciones climáticas, practicas utilizadas en el cultivo, madurez del fruto, métodos de cosecha y el manejo pos cosecha, entre otros (Dumas et al., 2003; Hamouz et al., 2010).

Varios autores han determinado la composición química y de compuestos bioactivos de variedades de sandías como es el caso de Tarazona-Díaz et al. (2011) quienes determinaron compuestos fenólicos, licopeno y citrulina en pulpa y corteza de 5 variedades de sandías (Fashion, Azabache, Motril, Kudam y Boston) cultivadas en Murcia (España), Davis et al. (2011) quienes evaluaron los niveles de citrulina en la pulpa de 56 variedades de sandía cultivadas en Lane, Oklahoma (Estados Unidos), Perkinz et al. (2002) determinó la composición química y contenido de citrulina en pulpa y corteza de 6 variedades de sandías ( 'Summer Flavour 800', 'Tri X 313', 'Orange Sunshine', 'Solid Gold', 'Orange Flesh Tendersweet' y 'Summer Gold') cultivadas al sudeste y sudoeste de Oklahoma (Estados Unidos), Fila et al. (2013) evaluó la composición química de tres especies de la Familia Cucurbitaceae, entre ellas la sandía (Citrullus lanatus), las cuales fueron obtenidas de un mercado público en Calabar (Nigeria) y Duran et al. (2017) quienes determinaron la composición química y contenido de citrulina de la corteza de sandías variedad Santa Amelia, obtenidas de 5 puntos de comercialización mayoristas ubicados en la ciudad de Valledupar (Colombia). Dada la necesidad de conocer la composición nutricional y de compuestos bioactivos de la sandía, el objetivo de esta investigación fue realizar una caracterización fisicoquímica, químico proximal, compuestos bioactivos y capacidad antioxidante a la pulpa y corteza de tres variedades de sandías (Citrullus lanatus) cultivadas en Cesar (Colombia). 


\section{MATERIALES Y MÉTODOS}

Los reactivos utilizados en este estudio fueron hidróxido de sodio (Merk, Darmstadt, Alemania), hexano (Merk, Darmstadt, Alemania), ácido clorhídrico (Merk, Darmstadt, Alemania), ácido sulfúrico (Panreac, Barcelona, España), ácido fosfórico (Mallinckrodt, Xalostoc, México), metanol (Panreac, Barcelona, España), ácido gálico (Merk, Hohenbrunn, Alemania), reactivo de Folin-Ciocalteu (Merk, Darmstadt, Alemania), carbonato de sodio (Merk, Darmstadt, Alemania), Trolox (Merk, Billerica, MA, EE.UU), radical libre DPPH (Merk, Billerica, MA, EE.UU), Diacetilmonoxima (monoxima 2,3-butanodiona, DAMO, Sigma Chemical Co., St. Louis, MO); tiosemicarbazida (TSC, Sigma Chemical Co.), L-citrulina (Sigma Chemical Co., St. Louis, EE.UU), entre otros, los cuales fueron de grado analítico.

El material vegetal utilizado en esta investigación fueron sandías (Citrullus lanatus) variedad Crimson Sweet y Jubilee fueron obtenidas de la finca La Oficina, ubicada en el municipio de Chimichagua, Colombia. Mientras que la variedad Santa Amelia fue obtenida de la finca San Carlos, municipio de Curumaní, Colombia. Las muestras fueron llevadas al laboratorio del Centro de Investigación para el Desarrollo de la Ingeniería (CIDI) de la Universidad Popular del Cesar (Valledupar, Colombia) donde se lavaron con agua para retirar materia extraña adherida a la superficie de la sandía y se desinfectaron con solución de hipoclorito de sodio (0.024 ppm de cloro libre residual). Se separó la pulpa de la corteza con un cuchillo de acero inoxidable y se empacaron individualmente en bolsas Ziploc $®$ y almacenadas a $-28^{\circ} \mathrm{C}$ para posteriores análisis (Durán et al., 2017).

\section{Caracterización fisicoquímica y químico proximal de la pulpa y corteza de sandía}

Se determinó pH (método 10.041/84 adaptado), acidez titulable expresada como ácido málico (método 942.15/90 adaptado), solidos solubles totales (método 932.12/90 adaptado), humedad (método 930.15/90 adaptado); cenizas (método 942.05/90 adaptado); extracto etéreo (método 920.39/90 adaptado); fibra cruda (método 962.09/90 adaptado); proteína cruda (Método Kjeldahl - 955.04/90 adaptado) acorde a la metodología descrita por la Asociación de Químicos Analíticos Oficiales (A.O.A.C, 2005). El contenido de carbohidratos se determinó por diferencia.

\section{Determinación de compuestos fenólicos totales y capacidad antioxidante de la pulpa y corteza de sandía}

Las muestras de pulpa y corteza fueron troceadas y licuadas en una licuadora Osterizer $\circledast$ (modelo 00465013-000) a la máxima velocidad por un tiempo de dos minutos. Después las muestras se secaron en un horno de secado convencional BIINDER $\AA$ a $60^{\circ} \mathrm{C}$. Las muestras ya secas se almacenaron en bolsas Ziploc $\AA$ en un desecador hasta su posterior uso. De las muestras almacenadas se pesaron $2.5 \mathrm{~g}$ de pulpa y corteza (de manera individual) en tubos falcon y se adicionaron $3 \mathrm{~mL}$ de metanol puro y se homogenizaron en un vortex por un minuto. Después, los tubos falcon fueron colocados en un baño de hielo $\left(4^{\circ} \mathrm{C}\right)$ y agitados a $200 \mathrm{rpm}$ por una hora a $9^{\circ} \mathrm{C}$. Inmediatamente, las muestras fueron centrifugadas a $1350 \mathrm{rpm}$ por 15 minutos a $4^{\circ} \mathrm{C}$. Por último se recuperó el sobrenadante, se filtró en papel filtro Wathman $\mathrm{N}^{\circ} 4$ y se almacenó el extracto metanolico filtrado a $4^{\circ} \mathrm{C}$ en frascos hasta que se realizaron las pruebas de compuestos fenólicos totales y capacidad antioxidante (Tarazona-Díaz et al., 2011).

Para la cuantificación de los compuestos fenólicos totales se utilizó el método colorimétrico de Folin Ciocalteu con algunas modificaciones (Tlili et al., 2011). Se tomó $125 \mu \mathrm{L}$ del extracto metanólico, se adicionó $500 \mu \mathrm{L}$ de agua destilada, $125 \mu \mathrm{L}$ de reactivo de Folin y se dejó reposar por tres minutos. Después, se adicionó $1250 \mu \mathrm{L}$ de carbonato de sodio (7\% p/v) y se aforó a $3000 \mu \mathrm{L}$ con agua destilada. Se dejó reposar en oscuridad por 90 minutos, pasado este tiempo se midió absorbancia a $768 \mathrm{~nm}$ y se compararon los resultados con una curva de ácido gálico. Los resultados se expresaron como mg ácido gálico equivalente (GAE)/ $100 \mathrm{~g}$ muestra fresca.

La capacidad antioxidante se determinó por el método del radical libre DPPH (2,2-Difenil-1-picrihidrazilo) con algunas modificaciones (Kuskoski et al., 2005). A $0.1 \mathrm{~mL}$ de extracto metanólico se le adicionaron $3.9 \mathrm{~mL}$ de una solución de $33 \mu \mathrm{M}$ de DPPH (previamente preparada en metanol). La mezcla se dejó reposar en oscuridad por 30 minutos, transcurrido este tiempo se midió la absorbancia a $522 \mathrm{~nm}$ y se compararon los resultados con una curva de Trolox. Los resultados fueron expresados como $\mu \mathrm{mol}$ Trolox equivalente (TEAC)/ $100 \mathrm{~g}$ de muestra fresca

\section{Determinación de citrulina de la pulpa y corteza de sandía}

En un tubo de $50 \mathrm{~mL}$ con un gramo de muestra seca (corteza o pulpa) se le adicionaron $40 \mathrm{~mL}$ de ácido acético $(0.1 \mathrm{M})$. Se agitó en vortex por un minuto y se centrifugó a $1350 \mathrm{rpm}$ por 15 minutos, e inmediatamente se colocaron los tubos en baño de hielo a $4^{\circ} \mathrm{C}$ durante 5 minutos. Se filtró con papel filtro 
№ 42 (Filtres Fiorini, Francia) y el extracto (filtrado) se almacenó en un frasco ámbar a $4{ }^{\circ} \mathrm{C}$ (Durán et al., 2017). Previamente a la determinación del contenido de citrulina se prepararon dos soluciones: ácido férrico (agua destilada: ácido sulfúrico: ácido fosfórico en una proporción de 55:25:20 v / v, con cloruro de férrico hexahidratado $250 \mathrm{mg} / \mathrm{L}$ ) (reactivo 1) y Diacetilmonoxima $(5 \mathrm{mg} / \mathrm{mL}$ ) (Reactivo 2). El reactivo cromogénico consistió en una mezcla de $50 \mathrm{~mL}$ de reactivo 2, $100 \mathrm{~mL}$ de reactivo 1 y $15 \mathrm{mg}$ de tiosemicarbazida. En un tubo de ensayo con $0.1 \mathrm{~mL}$ del extracto diluido (dilución 1:100) en una solución etanol: agua (2:1), se le adicionaron $3 \mathrm{~mL}$ del reactivo cromogénico, se agitó en vortex por un minuto y se llevó a un baño María a $100^{\circ} \mathrm{C}$ por 5 minutos y se enfrió a temperatura ambiente para medir la absorbancia a una longitud de onda de $530 \mathrm{~nm}$, y se cuantificó la citrulina mediante una curva de calibración con un patrón externo de L-citrulina. Los resultados se expresaron como $\mathrm{mg}$ citrulina/ $\mathrm{g}$ muestra fresca.

\section{Diseño experimental y análisis estadístico}

Para la caracterización fisicoquímica, químico proximal, de compuestos bioactivos y capacidad antioxidante se utilizó un diseño completamente al azar, donde los tratamientos fueron la pulpa y corteza de las variedades Crimson Sweet, Jubilee y Santa Amelia. La comparación de medias se realizó con la prueba de Tukey con un nivel de confianza del 95\%. El análisis de los datos obtenidos en esta investigación se realizó mediante un análisis de varianza (ANOVA) al 95\% utilizando el software estadístico STATGRAPHICS® Centurion XVI (StatPoint Technologies, Inc, Warrenton, VA, EE.UU).

\section{RESULTADOS Y DISCUSIÓN}

Los resultados se presentan y discuten en dos subsecciones: En una se presenta la caracterización fisicoquímica y químico proximal de la pulpa y corteza de sandía. En la segunda se muestra la determinación de compuestos fenólicos totales, capacidad antioxidante y citrulina de pulpa y corteza de sandía

\section{Caracterización fisicoquímica y químico proximal de la pulpa y corteza de sandía}

En la Tabla 1 se muestran los resultados obtenidos de la caracterización fisicoquímica de la pulpa y la corteza de las tres variedades de sandías estudiadas y en la Tabla 2 la caracterización químico proximal de las sandías. El porcentaje de acidez osciló entre $0.05 \%$ y $0.14 \%$, sin embargo no se presentó diferencias significativas entre corteza y pulpa en las diferentes variedades de sandía (Tabla 1). Estos valores fueron similares a los reportados por Tarazona-Díaz et al. (2011) quienes obtuvieron valores entre 0.107 y $0.134 \%$ para corteza, mientras que en pulpa la acidez estuvo entre 0.06 y 0.09 . El pH presentó diferencias significativas entre pulpa y corteza de una misma variedad, y entre las variedades estudiadas.

Tabla 1: Características fisicoquímicas de la pulpa y corteza de las sandías. Valores con letras (a,b,c,d,e) dentro de cada columna denota significancia en la prueba de Tukey $(p<0.05)$. Valores promedio de 3 repeticiones \pm desviación estándar

\begin{tabular}{|c|c|c|c|}
\hline Corteza & Acidez (\%) & $p H$ & Sólidos solubles ('Brix) \\
\hline Crimson Sweet & $0.13 \pm 0.04^{\mathrm{a}}$ & $5.43 \pm 0.05^{\mathrm{d}}$ & $5.0 \pm 0.5^{\mathrm{c}}$ \\
\hline Jubilee & $0.10 \pm 0.03^{\mathrm{a}}$ & $5.53 \pm 0.02^{\mathrm{cd}}$ & $3.6 \pm 0.3^{\mathrm{d}}$ \\
\hline Santa Amelia & $0.14 \pm 0.00^{\mathrm{a}}$ & $5.17 \pm 0.00^{\mathrm{e}}$ & $3.9 \pm 0.1^{\mathrm{d}}$ \\
\hline Pulpa & & & $7.9 \pm 0.3^{\mathrm{a}}$ \\
\hline Crimson Sweet & $0.09 \pm .00^{\mathrm{a}}$ & $5.68 \pm 0.05^{\mathrm{b}}$ & $6.6 \pm 0.3^{\mathrm{b}}$ \\
\hline Jubilee & $0.05 \pm 0.01^{\mathrm{a}}$ & $5.93 \pm 0.02^{\mathrm{a}}$ & $7.5 \pm 0.0^{\mathrm{ab}}$ \\
\hline Santa Amelia & $0.11 \pm 0.01^{\mathrm{a}}$ & $5.61 \pm 0.06^{\mathrm{bc}}$ & \\
\hline
\end{tabular}

En las cortezas, Santa Amelia presentó valores de pH estadísticamente inferiores a los reportados en Crimson Sweet y Jubilee; las cuales no presentaron diferencias entre sí ( $p>0.05)$. En las pulpas, Jubilee presentó valores de $\mathrm{pH}$ superiores $(\mathrm{p}<0.05)$ a los de Crimson Sweet y Santa Amelia, las cuales no presentaron diferencias significativas $(p>0.05)$ entre sí. En general el $\mathrm{pH}$ de la corteza fue un poco más ácido $(5.17-5.43)$ que el de las pulpas (5.61 - 5.93) contrario a lo reportado por Tarazona-Díaz et al. (2011) quienes no encontraron diferencias significativas de $\mathrm{pH}$ entre pulpa y corteza de los cultivares y entre cultivares estando estos entre 5.1 y 5.37 .

Con respecto al contenido de sólidos solubles totales ( ${ }^{\circ}$ Brix), la corteza de Crimson Sweet presentó mayor contenido de ${ }^{\circ}$ Brix $(p<0.05)$ que Jubilee y Santa Amelia, las cuales no presentaron diferencias significativas ( $p>0.05$ ) entre sí. Entre pulpas se presentó diferencias significativas entre Crimson Sweet y Jubilee, siendo mayor en esta primera. En general se reporta mayores contenido de sólidos solubles en las pulpas que en las cortezas (Perkins-Veazie et al., 2002;Tarazona-Díaz et al., 2011), esto debido a la presencia principalmente 
de mono y disacáridos tales como fructosa, glucosa y sacarosa (Kyriacou et al., 2018).Dichas diferencias y/o similitudes puede relacionarse a las características propias de cada cultivar entendiendo que son sandías diferentes, al igual que sus condiciones edafoclimáticas y agroecológicas (Durán et al., 2017).

Tabla 2: Caracterización químico proximal de la pulpa y corteza de las sandías. Valores con letras (a, b, c, d) indican significancia en la prueba de Tukey $(p<0.05)$. Valores promedio de 3 repeticiones \pm desviación estándar

\begin{tabular}{|c|c|c|c|c|c|c|}
\hline Corteza & $\begin{array}{c}\text { Humedad } \\
(\mathrm{g} / 100 \mathrm{~g} \\
\text { muestra } \\
\text { fresca) }\end{array}$ & $\begin{array}{c}\text { Ceniza } \\
(\mathrm{g} / 100 \mathrm{~g} \\
\text { muestra seca) }\end{array}$ & $\begin{array}{c}\text { Fibra } \\
(\mathrm{g} / 100 \mathrm{~g} \\
\text { muestra seca) })\end{array}$ & $\begin{array}{c}\text { Grasa } \\
(\mathrm{g} / 100 \mathrm{~g} \\
\text { muestra seca) }\end{array}$ & $\begin{array}{c}\text { Proteína } \\
(\mathrm{g} / 100 \mathrm{~g} \\
\text { muestra seca) }\end{array}$ & $\begin{array}{c}\text { CHO } \\
(\mathrm{g} / 100 \mathrm{~g} \\
\text { muestra seca) }\end{array}$ \\
\hline $\begin{array}{c}\text { Crimson } \\
\text { Sweet }\end{array}$ & $94.95 \pm 0.53^{\mathrm{a}}$ & $16.25 \pm 1.58^{\mathrm{ab}}$ & $12.24 \pm 1.08^{\mathrm{ab}}$ & $5.14 \pm 0.48^{\mathrm{a}}$ & $7.00 \pm 0.94^{\mathrm{c}}$ & $61.79 \pm 5.08^{\mathrm{cd}}$ \\
\hline Jubilee & $95.00 \pm 0.95^{\mathrm{a}}$ & $12.53 \pm 3.29^{\mathrm{b}}$ & $6.47 \pm 1.11^{\mathrm{ab}}$ & $5.50 \pm 0.49^{\mathrm{a}}$ & $7.76 \pm 2.00^{\mathrm{bc}}$ & $66.36 \pm 7.89^{\mathrm{bc}}$ \\
\hline $\begin{array}{c}\text { Santa } \\
\text { Amelia }\end{array}$ & $95.09 \pm 0.28^{\mathrm{a}}$ & $18.35 \pm 0.05^{\mathrm{a}}$ & $18.82 \pm 9.48^{\mathrm{a}}$ & $5.44 \pm 0.72^{\mathrm{a}}$ & $36.23 \pm 9.17^{\mathrm{a}}$ & $46.77 \pm 1.82^{\mathrm{d}}$ \\
\hline $\begin{array}{c}\text { Pulpa } \\
\text { Crimson } \\
\text { Sweet }\end{array}$ & $91.35 \pm 1.21^{\mathrm{b}}$ & $3.21 \pm 0.36^{\mathrm{c}}$ & $0.88 \pm 0.06^{\mathrm{b}}$ & $0.75 \pm 0.21^{\mathrm{b}}$ & $13.28 \pm 1.63^{\mathrm{bc}}$ & $84.24 \pm 5.23^{\mathrm{a}}$ \\
\hline $\begin{array}{c}\text { Jubilee } \\
\text { Sunta }\end{array}$ & $92.75 \pm 1.28^{\mathrm{ab}}$ & $1.90 \pm 0.19^{\mathrm{c}}$ & $0.61 \pm 0.02^{\mathrm{b}}$ & $0.69 \pm 0.08^{\mathrm{b}}$ & $17.85 \pm 3.03^{\mathrm{bc}}$ & $80.12 \pm 4.75^{\mathrm{ab}}$ \\
\hline $\begin{array}{c}\text { Smelia } \\
\text { Amant }\end{array}$ & $93.59 \pm 0.51^{\mathrm{ab}}$ & $4.16 \pm 0.24^{\mathrm{c}}$ & $1.07 \pm 0.10^{\mathrm{b}}$ & $0.90 \pm 0.14^{\mathrm{b}}$ & $23.18 \pm 2.55^{\mathrm{b}}$ & $70.71 \pm 2.20^{\mathrm{abc}}$ \\
\hline
\end{tabular}

El contenido de humedad no presentó diferencias significativas entre las cortezas de cada sandía, estos valores fueron cercanos al reportado por Tarazona-Díaz y Aguayo (2013) quienes hallaron una humedad en la corteza del $91.6 \%$ para el híbrido Fashion; sin embargo Fila et al. (2013) reportaron una humedad en la corteza de $67.75 \pm 0.64 \%$ diferencia que puede aludirse a que esta variedad es diferente a la de esta investigación. La humedad de las pulpas de la variedad Jubilee y el híbrido Santa Amelia no presentaron diferencias significativas $(p>0.05)$; la variedad Crimson Sweet fue la que presentó menor contenido de humedad en la pulpa en comparación con las otras sandías y muy similar al reportado por otra investigación (Fila et al., 2013). El contenido de ceniza de la corteza de Santa Amelia fue similar ( $p>0.05)$ al de Crimson Sweet, pero superior $(p<0.05)$ al de Jubilee, mientras que el contenido en las pulpas no presentaron diferencias significativas $(p>0.05)$ entre sí. Estas diferencias se pueden deberse a que el contenido de minerales varía según la variedad cultivada; ya que cada variedad responde de manera diferente al manejo poscosecha, condiciones de cultivo como también el tiempo de cosecha (Singh et al., 2016; Guil-Guerrero et al., 2006). En general, el comportamiento es similar al de otros autores donde el contenido de ceniza es mayor en la corteza que en la pulpa (Fila et al., 2013; Perkins-Veazie et al., 2002; Tarazona-Díaz y Aguayo, 2013).

El porcentaje de fibra cruda entre las cortezas no presentó diferencias significativas $(p>0.05)$. Esta misma tendencia se observó en las pulpas de las sandías estudiadas. Solo la variedad Santa Amelia tuvo un contenido de fibra mayor en corteza con respecto a la pulpa. Este comportamiento es acorde lo reportado por en la literatura, donde se evidencia que el contenido de fibra es superior en piel y corteza en comparación con la pulpa (Sagar et al., 2018). Los valores de fibra en pulpa fueron inferiores a los reportados por otros autores que fue del $1.25 \%$ (Fila et al., 2013), mientras que el contenido de fibra en corteza fue superior al reportado por Fila et al. (2013) que fue de $2.91 \%$. Con respecto al contenido de grasa, las cortezas presentaron mayores valores $(p<0.05)$ en comparación con las pulpas. El porcentaje de grasa de esta investigación fueron superior a los reportado por Fila et al. (2013) con valores de 0.21 y $0.38 \%$ para corteza y pulpa, respectivamente.

El contenido de proteína no presentó diferencias significativas ( $p>0.05)$ entre la corteza y la pulpa provenientes de las variedades Crimson Sweet y Jubilee, sin embargo, el contenido de proteína de la corteza Santa Amelia fue estadísticamente superior $(p<0.05)$ a las cortezas de Crimson Sweet y Jubilee, las cuales no presentaron diferencias $(p>0.05)$ entre sí. El contenido de proteína no presentó diferencias significativas $(p<0.05)$ entre las variedades estudiadas. Tarazona y Aguayo (2013) reportan un contenido de proteína $16.27 \%$ en la corteza del híbrido Fashion, el cual es inferior al encontrado en la corteza del híbrido Santa Amelia en esta investigación, pero superior al reportado en Crimson Sweet y Jubilee. Por otro lado, Fila et al. (2013) reportaron porcentajes de proteína en pulpa $(0.83 \%)$ y corteza $(7.11 \%)$, los cuales fueron inferiores a los hallados en esta investigación. Perkinz-Veazie et al. (2002) obtuvieron valores de proteína en pulpa y corteza de 9.16 y $9.89 \%$, respectivamente. El contenido de carbohidratos en la pulpa no presentó diferencias significativas $(p<0.05)$ entre las variedades estudiadas, mientras que en las cortezas, Jubilee tuvo mayor 
contenido de carbohidratos $(p<0.05)$ que Santa Amelia, pero similar a Crimson Sweet $(p<0.05)$. En general, el contenido de carbohidratos fue mayor en pulpa que en corteza, esto se puede apreciar al observar los 'Brix de las pulpa, los cuales fueron mayores que las corteza, indicando mayor presencia de azúcares como glucosa, fructosa y sacarosa en las pulpas (Perkins-Veazie et al., 2002; Tarazona-Díaz et al., 2011; Kyriacou et al., 2018)

\section{Compuestos fenólicos totales, capacidad antioxidante y citrulina de pulpa y corteza de sandía}

En la Tabla 3 se muestra el contenido de compuestos fenólicos totales, la capacidad antioxidante y el contenido de citrulina de las sandías. No se encontraron diferencias significativas $(p>0.05)$ en la capacidad antioxidante (CA) entre las cortezas de las sandías. Sin embargo, las pulpas de las sandías presentaron diferencias significativas $(p<0.05)$, siendo la CA mayor en la Santa Amelia, seguida de Jubilee y Crimson Sweet.

Tabla 3: Compuestos fenólicos totales, citrulina y capacidad antioxidante de la pulpa y corteza de las sandías. Valores con letras $(a, b, c, d)$ dentro de cada columna denota significancia en la prueba de Tukey $(p<0.05)$, valores promedio de 3 repeticiones \pm desviación estándar.

\begin{tabular}{|c|c|c|c|}
\hline Corteza & $\begin{array}{c}\text { Capacidad antioxidante } \\
(\mu \text { mol TEAC/ } 100 \mathrm{~g} \\
\text { muestra fresca) }\end{array}$ & $\begin{array}{c}\text { Compuestos fenólicos totales } \\
(\mathrm{mg} \mathrm{GAE} / 100 \text { muestra fresca) }\end{array}$ & $\begin{array}{c}\text { Citrulina } \\
\text { (mg/ g muestra fresca) }\end{array}$ \\
\hline Crimson Sweet & $361.96 \pm 8.87^{\mathrm{c}}$ & $15.19 \pm 9.61^{\mathrm{c}}$ & $0.46 \pm 0.06^{\mathrm{d}}$ \\
\hline Jubilee & $365.15 \pm 30.57^{\mathrm{c}}$ & $21.52 \pm 16.71^{\mathrm{bc}}$ & $2.41 \pm 0.03^{\mathrm{e}}$ \\
\hline Santa Amelia & $325.30 \pm 15.08^{\mathrm{c}}$ & $7.61 \pm 5.21^{\mathrm{c}}$ & $0.85 \pm 0.10^{\mathrm{c}}$ \\
\hline Pulpa & \\
\hline \multicolumn{3}{|l|}{} \\
\hline Crimson Sweet & $217.69 \pm 33.67^{\mathrm{d}}$ & $61.82 \pm 2.24^{\mathrm{a}}$ & $0.42 \pm 0.02^{\mathrm{d}}$ \\
\hline Jubilee & $505.28 \pm 4.90^{\mathrm{b}}$ & $55.64 \pm 25.88^{\mathrm{ab}}$ & $1.11 \pm 0.14^{\mathrm{b}}$ \\
\hline Santa Amelia & $869.30 \pm 42.40^{\mathrm{a}}$ & $33.47 \pm 10.49^{\mathrm{abc}}$ & \\
\hline
\end{tabular}

La CA de las pulpas de esta investigación tuvieron valores superiores a las variedades estudiadas por Tlili et al. (2011) que fueron Dumara $(205.5 \mu \mathrm{mol}$ TEAC/ $100 \mathrm{~g})$, P403 (242.9 $\mu \mathrm{mol}$ TEAC/ $100 \mathrm{~g})$, P503 (182.4 $\mu \mathrm{mol}$ TEAC/ $100 \mathrm{~g})$ y Aramis $(225.7 \mu \mathrm{mol}$ TEAC/ $100 \mathrm{~g})$, sin embargo la CA de Crimson Sweet $(290.9 \mu \mathrm{mol}$ TEAC/ $100 \mathrm{~g})$ y Giza $(271.3 \mu \mathrm{mol}$ TEAC/ $100 \mathrm{~g})$ fueron superiores a la CA de Crimson Sweet de esta investigación. Por otro lado, Zapata et al. (2013) reportaron una mayor CA en pulpa de guayaba agria cultivada en la ciudad de Montería (Colombia) con un valor de $1177.89 \mu$ mol TEAC/ $100 \mathrm{~g}$ de muestra fresca. El contenido de compuestos fenólicos totales (CFT) en pulpa y corteza de las sandías presentaron diferencias significativas $(p<0.05)$, hallándose un mayor contenido de CFT en pulpa que en corteza. El contenido de CFT en las pulpas presentó ligeras diferencias, teniendo mayor contenido Crimson Sweet, seguido de Jubilee y Santa Amelia. Mientras que las cortezas, el comportamiento observado fue diferente ya que el contenido de CFT fue superior en Jubilee, seguido de Crimson Sweet, y Santa Amelia. El contenido de CFT en pulpa hallados en esta investigación fueron superiores a los hallados por Tlili et al. (2011) con valores entre 8.90 y $14.73 \mathrm{mg}$ GAE/100 g de muestra pulpa fresca, pero inferiores a los obtenidos por Perkins-Veazie et al. (2002) quienes reportaron contenidos de compuestos fenolicos en sandías Summer Flavor 800 y Tri-X-313 de 87.21 y 91.82 mg GAE/100 g muestra fresca, respectivamente, y por Denardin et al. (2015) reportaron valores superiores expresados en muestra fresca de 6 frutas nativas de Brasil: arazá rojo (660.19 mg GAE/100 g), pitanga naranja (457.43 mg GAE/100 g), pitanga roja (433.84 mg GAE/100 g), pitanga morada (799.80 mg GAE/100 g), mora Xavante (816.50 mg GAE/100 g) y mora Cheroke (718.65 mg GAE/100 g).

En general, los compuestos fenólicos son productos secundarios del metabolismo de la plantas. La síntesis de estos compuestos están influenciado por factores como infecciones, lesiones, radiación UV, salinidad del suelo, calor, genotipo, tipo de fruta, tipo de suelo, clima, grado de madurez, condiciones del cultivo, tiempo de cosecha, manipulación pos cosecha, entre otros factores mientras que la actividad antioxidante está más influenciada por la presencia e interacción de los diferentes compuestos fenólicos con capacidad antioxidante tales como licopeno, vitamina C, citrulina, ácidos clorogénicos, entre otros (Singh et al., 2016; Silva et al., 2014;Denardin et al., 2015; Dumas et al., 2003; Hamouz et al., 2010; Pérez-López et al., 2007; Pincemail et al., 2012; Soteriou et al.,2014;Tarazona-Díaz et al., 2011; Tlili et al., 2011).

El contenido de Citrulina en corteza y pulpa, respectivamente presentó diferencias significativas $(p<0.05)$ entre variedades (ver Tabla 3.). En pulpa y corteza, el contenido de Citrulina en Santa Amelia fue estadísticamente mayor $(p<0.05)$ al de Crimson Sweet y Jubilee, respectivamente. El contenido de Citrulina en la corteza de Crimson Sweet y Jubilee fue inferior a los hallados por otros autores con valores de 2.0 $7.2 \mathrm{mg} / \mathrm{g}$ de muestra fresca (Tarazona-Díaz et al., 2011) y $0.8-1.5 \mathrm{mg} / \mathrm{g}$ muestra fresca (Rimando y PerkinsVeazie, 2005); pero similares a los hallados por Durán et al. (2017) de $0.185-0.645 \mathrm{mg} / \mathrm{g}$ muestra fresca. 
La corteza de Santa Amelia presentó el mayor contenido de Citrulina respecto a las demás sandías evaluadas obteniendo concentraciones cercanas a lo reportado por los autores mencionados anteriormente. En la pulpa, los contenidos de Crimson Sweet y Jubilee fueron menores a los hallados por Tarazona-Díaz et al. (2011) de $1.1-4.7 \mathrm{mg} / \mathrm{g}$ muestra fresca Rimando y Perkins-Veazie (2005) de $1.0-3.5 \mathrm{mg} / \mathrm{g}$ muestra fresca y Davis et al. (2011) de 1.09- $4.52 \mathrm{mg} / \mathrm{g}$ muestra fresca. Sin embargo, los contenidos de Santa Amelia fueron similares al compararlos con los obtenidos por los mismos autores. Las diferencias entre el contenido de citrulina de pulpa y corteza de las sandías estudiadas y citadas pueden deberse a las condiciones medioambientales (humedad relativa, temperatura del aire), condiciones del suelo (temperatura, humedad y fertilidad), genotipo de la variedad humedad relativa y temperatura del aire, como también la temperatura, humedad y fertilidad del suelo (Davis et al., 2011;Tarazona-Díaz et al., 2011). Además, la acción de los aminoácidos sobre el organismo vegetal siempre se ha centrado en su capacidad para ayudarlos a superar situaciones de estrés y situaciones de gran actividad metabólica como las que se producen en la germinación, senescencia floral hasta la maduración del fruto que intervienen en la regulación de procesos fisiológicos fundamentales como el equilibrio hídrico de la planta por condiciones climáticas desfavorables (Hildebrandt et al., 2015; Kyriacou et al., 2018).

\section{CONCLUSIONES}

Dado el alto contenido de humedad (>94,00\%) y el bajo $\mathrm{pH}(5.17-5.43)$ de las cortezas estudiadas, en particular la corteza de Santa Amelia, la cual tuvo contenidos superiores de ceniza, fibra, grasa, proteína y citrulina, hace necesario el uso de tratamientos de procesamiento y conservación adecuados para el aprovechamiento tanto de los nutrientes como de los compuestos bioactivos presentes en este residuo agroindustrial; los cuales pueden ser degradados por la acción de microrganismos tales como hongos y bacterias. La aplicación de estos tratamientos permitiría la incorporación de estos compuestos en matrices alimentarias aprovechando así sus beneficios para la salud y de paso disminuiría el impacto ambiental generado por estos residuos.

\section{AGRADECIMIENTOS}

Los autores agradecen a la Vicerrectoría de Investigación y Extensión de la Universidad Popular del Cesar por la financiación de este proyecto (Acuerdo 059 del 19 de Abril del 2016) y al Centro de Investigación para el Desarrollo de la Ingeniería (CIDI) por facilitar el uso de su laboratorio para la ejecución de la fase experimental de esta investigación.

\section{REFERENCIAS}

A.O.A.C, Association of Official Analytical Chemists, Official methods of analysis of the AOAC International, $18^{\mathrm{a}}$ edición. H. W. y G. Latimer, Eds.. Gaithersburg, MD,Estados Unidos (2005)

Al-Sayed, H. M. A., y A.R. Ahmed, Utilization of Watermelon Rinds and Sharlyn Melon Peels as a Natural Source of Dietary Fiber and Antioxidants in Cake, doi:10.1016/J.AOAS.2013.01.012, Annals of Agricultural Sciences , 58(1), 83-95 (2013)

Cohen-Manrique, C. S., J. A Rodríguez-Manrique y R. D. Salgado-Ordosgoitia, Modelado del Microclima de un Cultivo de Sandía (Citrullus lanatus) en la Sub-región Sabana del Departamento de Sucre, Colombia., doi:/10.4067/S071807642018000500335, Información Tecnológica, 29(5), 335-344 (2018)

Davis, A. R., C.L. Webbner III y otros cuatro autores, L-citruline Levels in Watermelon Cultigens Tested in Two Environments. HortScience, ISSN:2327-9834, 46(12), 1572-1575 (2011)

Denardin, C.C., G. E. Hirsh y otros seis autores, Antioxidant Capacity and Bioactive Compounds of four Brazilian Native Fruits, doi: 10.1016/J.JFDA.2015.01.006, Journal of Food and Drug Analysis, 23(3), 387-398 (2015)

Dumas, Y., M. Dadomo y otros dos autores, Effects of Environmental Factors and Agricultural Techniques on Antioxidant Content of Tomatoes, doi:10.1002/jsfa.1370, Journal of the Science of Food and Agriculture, 83(5), 369-382 (2003)

Durán, R., M.E. Villegas. y I. Nieves, Caracterización y Extracción de Citrulina de Corteza de Sandía (Citrullus lanatus"thunb") Consumida en Valledupar, TEMAS AGRARIOS, ISSN:2389-9182, 22(1), 62-69 (2017)

Fila, W. A., E. H. Itam y otros cinco autores, Comparative Proximate Compositions of Watermelon Citrullus Lanatus, Squash Cucurbita Pepo'I and Rambutan Nephelium Lappaceum, International Journal of Science and Technology, ISSN: 2049-7318, 2(1), 81-88 (2013)

Guil-Guerrero, J. L., C. Martínez-Guirado y otros dos autores, Nutrient Composition and Antioxidant Activity of 10 Pepper (Capsicum annuun) Varieties, doi:10.1007/s00217-006-0281-5, European Food Research and Technology, 224(1), 1-9 (2006)

Hamouz, K., J. Lachman y otros cuatro autores, Effect of Natural and Growing Conditions on the Content of Phenolics in Potatoes with Different Flesh Colour, doi:10.17221/49/2010-PSE, Plant, Soil and Environment, 56(8), 368-374 (2010) 
Hildebrandt, T. M., A. Nunes y otros dos autores, Amino Acid Catabolism in Plants, doi.org/10.1016/j.molp.2015.09.005, Molecular Plant, 8(11), 1563-1579 (2015)

Kaore, S. y N. Kaore, Citrulline: Pharmacological Perspectives and Role as a Biomarker in Diseases and Toxicities, doi: 10.1016/B978-0-12-404630-6.00053-1, Biomarkers in Toxicology, 883-905 (2014)

Koocheki, A., S. Razavi y otros cuatro autores, Physical Properties of Watermelon Seed as a Function of Moisture Content and Variety, International Agrophysics, ISSN:0236-8722, 21(4), 349-359 (2007)

Kuskoski, E. M., A. Asuero y otros tres autores, Aplicación de Diversos Métodos Químicos para Determinar Actividad Antioxidante en Pulpa de Frutos, doi:10.1590/S0101-20612005000400016, Ciência e Tecnologia de Alimentos, 25(4), 726-732 (2005)

Kyriacou, M. C., D. I. Leskovar, y otros dos autores, Watermelon and Melon Fruit Quality: The Genotypic and Agroenvironmental Factors Implicated, doi: 10.1016/J.SCIENTA.2018.01.032, Scientia Horticulturae, 234, $393-408$ (2018)

Mori, A.,M. Morita y otros cuatro autores L-Citrulline Dilates Rat Retinal Arterioles Via Nitric Oxide- and Prostaglandindependent Pathways In Vivo, doi:10.1016/J.JPHS.2015.02.012, Journal of Pharmacological Sciences, 127(4), 419-423 (2015)

Pérez-López, A. J., F. M del Amor y otros tres autores, Influence of Agricultural Practices on the Quality of Sweet Pepper Fruits as Affected by the Maturity Stage, doi:10.1002/jsfa.2966, Journal of the Science of Food and Agriculture, 87(11), 2075-2080 (2007)

Perkins-Veazie, P. M., N. Maness y R. Roduner, Composition of Orange,Yellow and Red-fleshed Watermelons. Cucurbitaceae, 436-440 (2002) Tomado de Tlili et al. (2011)

Pincemail, J., C. Kevers y otros tres autores, Cultivars, Culture Conditions, and Harvest Time Influence Phenolic and Ascorbic Acid Contents and Antioxidant Capacity of Strawberry (Fragaria $x$ ananassa), doi:10.1111/j.17503841.2011.02539.x, Journal of Food Science, 77(2), C205-C210 (2012)

Rimando, A. M., y P. M. Perkins-Veazie, Determination of Citrulline in Watermelon Rind, doi:10.1016/J.CHROMA.2005.05.009, Journal of Chromatography A, 1078(1-2), 196-200 (2005)

Sagar, N. A., S. Pareek y otros tres autores, Fruit and Vegetable Waste: Bioactive Compounds, Their Extraction, and Possible Utilization, doi: 10.1111/1541-4337.12330, Comprehensive Reviews in Food Science and Food Safety, 17(3), 512-531 (2018)

Silva, L.M.R. da, E. A .T de Figueredo y otros cinco autores, Quantification of Bioactive Compounds in Pulps and Byproducts of Tropical Fruits from Brazil, doi: 10.1016/J.FOODCHEM.2013.08.001, Food Chemistry, 143, 398-404 (2014)

Singh, J. P., A. Kaur y otros dos autores, Composition, Bioactive Compounds and Antioxidant Activity of Common Indian Fruits and Vegetables, doi: 10.1007/s13197-016-2412-8, Journal of Food Science and Technology, 53(11), 4056-4066 (2016)

Soteriou, G. A., M. C. Kyriacou y otros dos autores, Evolution of Watermelon Fruit Physicochemical and Phytochemical Composition during Ripening as Affected by Grafting, doi: 10.1016/J.FOODCHEM.2014.04.120, Food Chemistry, 165, 282-289 (2014)

Tarazona-Díaz, M. P. , A. Sánchez y E. Aguayo, Preservation of Bioactive Compounds and Quality Parameters of Watermelon Juice Enriched with L-Citrulline through Short Thermal Treatment, doi: 10.1155/2017/3283054, Journal of Food Quality, 1-10 (2017)

Tarazona-Díaz, M. y E. Aguayo, Assessment of By-products from Fresh-cut Products for Reuse as Bioactive Compounds, doi:10.1177/1082013212455346, Food Science and Technology International, 19(5), $439-446$ (2013)

Tarazona-Díaz, M. P., J. Viegas y otros dos autores, Bioactive Compounds From Flesh and By-product of Fresh-cut Watermelon Cultivars, doi:10.1002/jsfa.425, Journal of the Science of Food and Agriculture, 91(5), 805-812 (2011)

Tlili, I., C. Hdider y otros cuatro autores, Bioactive Compounds and Antioxidant Activities of Different Watermelon (Citrullus lanatus (Thunb.) Mansfeld) Cultivars as Affected by Fruit Sampling Area, doi:10.1016/J.JFCA.2010.06.005, Journal of Food Composition and Analysis, 24(3), 307-314 (2011)

Zapata, K., F. B., Cortes y B. A. Rojano, Polifenoles y Actividad Antioxidante del Fruto de Guayaba Agria (Psidium araca), doi: 10.4067/S0718-0764201300050001, Información Tecnológica, 24(5), 103-112 (2013) 